\title{
Patrones de variabilidad ambiental en el mar peruano
}

\author{
Environmental variability patterns in the Peruvian waters
}

Roberto Flores, Marco Espino, Gertrudes Luque y Jorge Quispe

Instituto del Mar del Perú, esquina Gamarra y General Valle S/N Chucuito, Callao, Perú.

Email Roberto Flores: rflores@imarpe.gob.pe
Citación:

Flores R., M. Espino, G. Luque \& J. Quispe. 2013. Patrones de variabilidad ambiental en el mar peruano. En: Csirke J., R. Guevara-Carrasco \& M. Espino (Eds.). Ecología, pesquería y conservación del jurel (Trachurus murphyi) en el Perú. Rev. peru. biol. número especial 20(1): 021 - 028 (Septiembre 2013)

\section{Resumen}

Se describen los escenarios ambientales y los patrones de variabilidad observados en el mar peruano a partir del análisis de información obtenida por el Instituto del Mar del Perú entre los años 1960 - 2011. Se examinan datos de cruceros de investigación oceanográfica, de evaluación de recursos pesqueros y bío-oceanográficos, además de información de diversas publicaciones sobre el mar peruano y sus recursos. Los datos de temperatura, salinidad y contenido de oxígeno, fueron interpolados para obtener promedios mensuales y construir series espaciales y temporales. En la superficie, las Aguas Costeras Frías (ACF) y Aguas Subtropicales Superficiales (ASS) crean condiciones ambientales diferentes a las de la parte subsuperficial donde predomina la intromisión de las aguas ecuatoriales a través de la Corriente Subsuperficial Perú-Chile (CSPC). En el norte, durante el verano y otoño, predominan en la capa superficial aguas cálidas de origen ecuatorial. En el centro, aguas de origen ecuatorial interactúan con las ACF del afloramiento costero. El sur está influenciado por las ASS y el afloramiento costero con mayor intensidad, indicando una mayor actividad en el Sistema de la Corriente del Perú (SCP).

Palabras clave: Escenarios ambientales, Perú, ACF, ASS, CSPC

\section{Abstract}

Environmental scenarios and variability patterns observed in the Peruvian waters are described based on the analysis of information obtained by the Instituto del Mar del Peru between 1960 and 2011. This information includes data from oceanographic, stock assessment and bio-oceanographic scientific research surveys, published information was also used to complete this study. Sea temperature, salinity and oxygen content data was interpolated to obtain monthly averages and build temporal and spatial series. Surface Cold Coastal Waters (ACF) and Subtropical Surface Water (SSW) create environmental conditions that are different from those in the subsurface, where the intrusion of equatorial waters dominates through the Peru-Chile Subsurface Current (CSPC). In the north, during the summer and fall, there is predominance in the surface layer of waters from the equatorial system. In the central zone the equatorial waters interact with ACF from coastal upwelling. The south is more strongly influenced by the coastal upwelling and the ASS, which is an indicator of increased activity in the Current System of Peru (SCP).

Keywords: Environmental scenarios, Peru, ACF, ASS, CSPC

\section{Introducción}

Frente a la costa occidental de Sudamérica se encuentra una de las áreas de mayor productividad biológica, que influencia todos los niveles tróficos del ecosistema marino. El área marítima frente a la costa del Perú representa $0.1 \%$ del área oceánica del planeta y produce más del $10 \%$ del total de capturas de peces a nivel mundial (Chávez et al. 2008). Esta alta productividad biológica se debe principalmente a las condiciones físicas muy especiales existentes en el Sistema de la Corriente del Perú (SCP) y en particular a la presencia de celdas de afloramiento costero, y a la dinámica oceánica (Zuta \& Guillén 1970, Chávez et al. 1989, Bakun \& Sweeks 2008, Graco et al. 2007).

Cerca de la costa peruana, y a lo largo de ésta, los vientos dominantes hacia el ecuador y el rotacional del esfuerzo del viento ciclónico dan lugar a intensas celdas de 
afloramiento caracterizadas por una franja de aguas frías (Fig. 1a) y de alta productividad biológica. Este régimen de viento costero permanente conduce a un sistema típico de corriente de afloramiento compuesto por flujos superficiales hacia el ecuador y flujos subsuperficiales hacia el polo. La circulación superficial hacia el ecuador está compuesta por la Corriente Costera Peruana (CCP) y la Corriente Oceánica Peruana (COP) (Fig. 1b). Estas corrientes cerca a la superficie están principalmente impulsadas por el viento. Sin embargo, la CCP es reforzada a través del ajuste geostrófico asociado con fuertes gradientes de temperatura y densidad (Fig. 1a). La circulación subsuperficial hacia el Polo está principalmente compuesta por la Corriente Subsuperficial Perú-Chile (CSPC) a lo largo de la pendiente y plataforma continental. En la parte norte es reforzada con la Corriente Subsuperficial Ecuatorial (CSE) y la Contracorriente Subsuperficial primaria (CCSSp). Éstas corrientes subsuperficiales son capaces de alcanzar la costa peruana, mientras que más al sur la CSPC recibe el aporte adicional de otro flujo ecuatorial subsuperficial conocido como la Contracorriente Subsuperficial secundaria (CCSSs) o Jet Tsuchiya secundaria, (Fig. 1a) (Montes et al 2010, Chaigneau et al 2013).

En el presente trabajo, se describen los escenarios ambientales existentes en el mar peruano y los patrones de variabilidad imperantes, a partir del análisis de información obtenida in situ en los últimos 50 ańos por el Instituto del Mar del Perú (IMAR$\mathrm{PE}$ ) en sus cruceros de investigación oceanográfica, cruceros de evaluación de recursos pesqueros, cruceros de investigación internacionales, y otras publicaciones orientadas al conocimiento del mar peruano y sus recursos.

\section{Material y métodos}

Se recopilaron datos de temperatura, salinidad y contenido de oxígeno, del periodo 1960 - 2011, de 256 cruceros de investigación nacional e internacional, totalizando 8959 perfiles verticales distribuidos entre los 04 y $18^{\circ} \mathrm{S}$. Así mismo se incluye información de prospecciones biológicas, oceanográficas y pesqueras realizadas en el mismo periodo. En la capa superficial de $0-30 \mathrm{~m}$ de profundidad entre las $50-100 \mathrm{mn}$ de distancia se obtuvo la climatología mensual y estacional de la temperatura.

Para la determinación de la profundidad de la isoterma de 15 ${ }^{\circ} \mathrm{C}(1961$ - 2011) se trabajó información obtenida de perfiles hasta los $300 \mathrm{~m}$ de profundidad entre los 05 y $18^{\circ} \mathrm{S}$, que se separaron en 3 zonas: norte $\left(05-08^{\circ} \mathrm{S}\right)$, centro $\left(08-13^{\circ} \mathrm{S}\right)$ y sur $\left(13-18^{\circ} \mathrm{S}\right)$, entre las $50-100 \mathrm{mn}$ de la costa. Usando el método de estimador geoestadístico kriging se completaron los datos faltantes y se interpolaron por cada metro hasta los 300 m de profundidad (Bardossy et al. 1988, Bourgault \& Marcotte 1991, Journel \& Huijbrenchts 1978), y luego se extrajeron los valores de la profundidad de la isoterma de $15^{\circ} \mathrm{C}\left(\mathrm{Z}_{15^{\circ} \mathrm{C}}\right)$ con un error menor a un metro. También se calcularon promedios mensuales y promedios simples de todas las estaciones del ańo, desde 1961 hasta 2011

Todas las series de la $Z_{15}{ }^{\circ} \mathrm{C}$ se filtraron con un promedio móvil de 5 meses centrado en 2 meses. Es decir, para cada mes se calculó el promedio incluyendo los 2 meses antes y 2 meses después. Las series obtenidas se promediaron de manera simple y se obtuvo una serie única de la $\mathrm{Z}_{15^{\circ} \mathrm{C}}$.

Para evaluar las condiciones de corrientes marinas a lo largo de la costa peruana se aplicó la metodología de procesamiento empleada por Chaigneau et al. (2013). Los perfiles verticales y distribución de las corrientes horizontales fueron obtenidos en los cruceros realizados por el IMARPE utilizando el BIC José Olaya, entre los años 2008 y 2012, con un Perfilador de Corrientes Acústico Doppler (ADCP) marca RD Instrumentos $75 \mathrm{kHz}$ de banda ancha, montado en el casco del barco. Para evitar la interferencia entre el ADCP y la Ecosonda Simrad de doble-frecuencia EK60, se utilizó un sistema sincronizador.

Los datos del ADCP fueron procesados y calibrados utilizando el sistema estándar Common Ocean Data Access (CODAS) desarrollado por la Universidad de Hawaii. Se hicieron pruebas a los datos para obtener seńales de retornos con errores razonables. Las velocidades relativas fueron rotadas desde el transductor respecto a la referencia de la Tierra usando el Girocompás del barco. Para determinar la velocidad absoluta de la corriente, se utilizó el Girocompás a bordo y el Sistema de Posicionamiento Global (GPS). Para el ancho de celda de 30 minutos se utilizó un filtro de paso bajo para las velocidades en la capa de referencia de 50 a $180 \mathrm{~m}$. Finalmente los resultados corresponden a promedios interpolados cada $10 \mathrm{~m}$ entre 25 a $600 \mathrm{~m}$ (Chaigneau et al. 2013).

\section{Resultados y discusión}

Sobre la base de datos e información descritos por muchos autores y con información procesada para este artículo, es posible caracterizar los siguientes escenarios ambientales y los siguientes patrones de variabilidad en el mar peruano.

Escenarios ambientales en el Sistema de la Corriente del Perú.- El mar peruano presenta las siguientes características (Fig. 1):

- Una extensa área de afloramiento costero, que normalmente puede abarcar de 0 a 30 millas de la costa en promedio (Morón 2000), pudiendo extenderse a más o menos, en función de la estación del año (estacionalidad), de los procesos derivados del ENSO (interanual), de la oscilación decadal (periódicos) y la secularidad (Espino 2003, Gaxiola et al. 2008, Espino \& Yamashiro 2012).

- Una reducida área influenciada por las Aguas Tropicales Superficiales al norte de los $04^{\circ} \mathrm{S}$, donde encontramos una alta biodiversidad y bajas biomasas.

- Esto deja una zona de mezcla entre los 04 y los $06^{\circ} \mathrm{S}$, con influencia alternada del sur y el norte, dependiendo de la estación imperante del año. En el invierno-primavera prevalecen condiciones del sur y en el verano-otoño condiciones del norte. Esta zona también se caracteriza por una alta biodiversidad, pero menor a la anterior y con bajas biomasas.

- Por fuera de las zonas de afloramiento costero, se localizan las áreas oceánicas dominadas por las Aguas Subtropicales Superficiales (ASS) de condiciones cálidas y que se acercan a la zona costera con mayor intensidad en los veranos y otoños, pero que son influenciadas e inclusive desplazadas por las aguas costeras de afloramiento, durante los inviernos y primaveras. En estas áreas, en la columna de agua que va desde la superficie hasta profundidades mayores a los 1000 metros, domina la Vinciguerria lucetia que constituye alimento de atunes Thunnus spp., dorados Coryphaena hippurus y calamares Dosidicus gigas (Rosas Luis 2007) y 

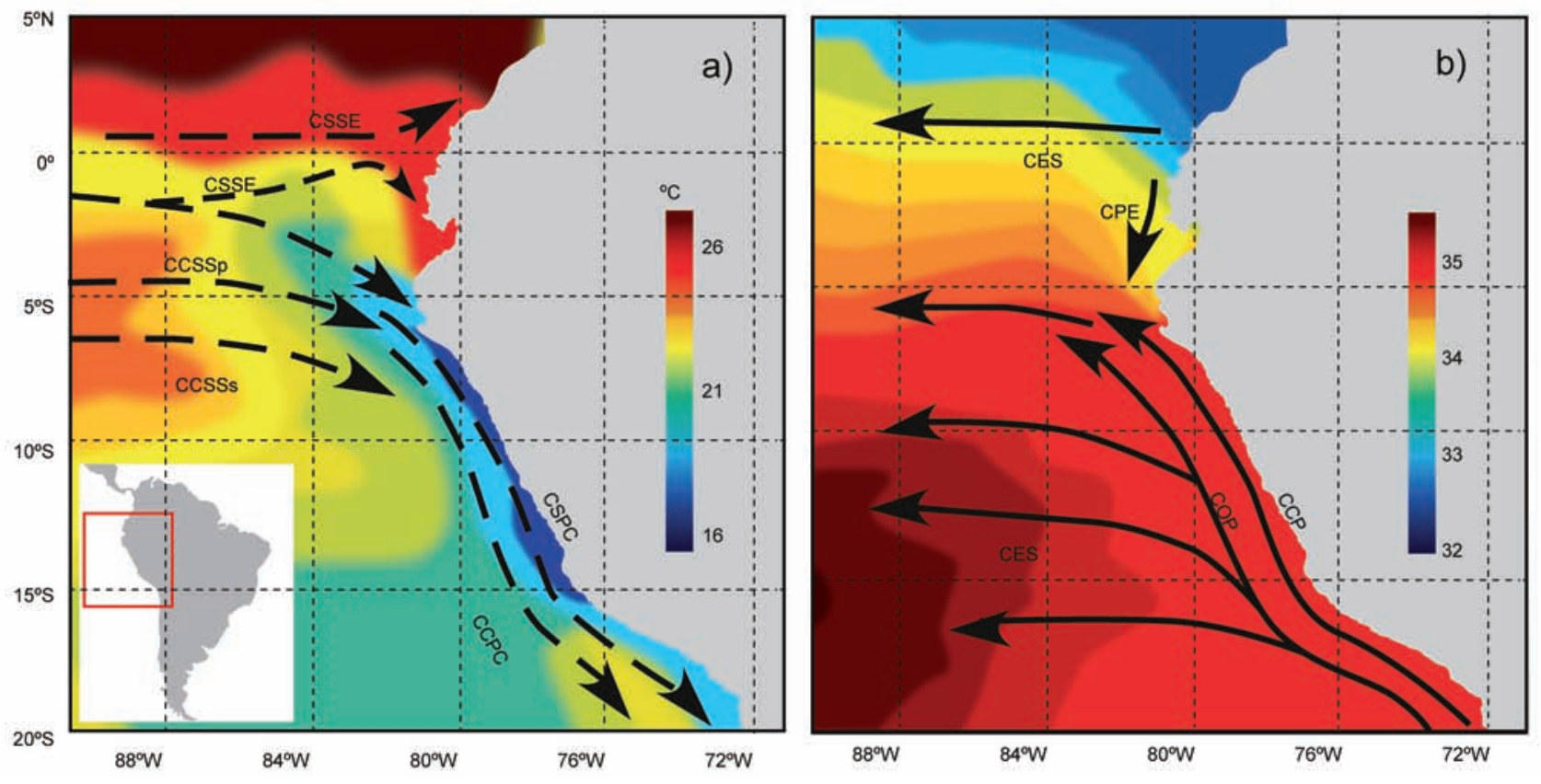

Figura 1. Sistema de la Corriente del Perú (SCP), a) corrientes subsuperficiales, (b) corrientes superficiales. (Chaigneau et al. 2013) Figure 1. Peruvian Current System (PCS), a) subsurface currents, b) surface currents. (Chaigneau et al. 2013)

también del jurel T. murphy. Al respecto, Alegre et al. (2013) plantean que fuera de las $130 \mathrm{mn}$ de la costa la presencia de peces mesopelágicos se incrementa ligeramente en la dieta de T. murphy; sin embargo, existe poca información disponible de pesquería de T. murphy a distancias mayores a $150 \mathrm{mn}$ donde la abundancia de peces mesopelágicos como la Vinciguerria lucetia es mayor. Cabe destacar asimismo que durante la última década, la mayor abundancia de $V$. lucetia se localizó al sur de los $16^{\circ} \mathrm{S}$, siendo en dicha zona donde se han realizado las mayores capturas de T. murphy (Gutiérrez Torero 2002).

Subsuperficialmente, por el norte se identifican los siguientes subsistemas:

- La zona comprendida entre los $3^{\circ} 30^{\prime} \mathrm{S}$ hasta los $6^{\circ} \mathrm{S}$ está influenciada por la presencia de la CSE y la CCSSp, estas dos corrientes ecuatoriales alcanzan la costa peruana y contribuyen con la parte norte de la CSPC.

- $\mathrm{Al}$ sur de los $6^{\circ} \mathrm{S}$ la CSPC recibe aporte adicional de otro flujo ecuatorial subsuperficial (CCSSs) o Jet Tsuchiya secundario, que se desplaza hasta más allá de los $40^{\circ} \mathrm{S}$.

Con información de cruceros del verano del 2011 y primavera del 2012, se determinó que los flujos superficiales están modulados por la presencia de giros anticiclónicos (Fig. 2a), formando frentes asociados a vorticidades del orden del Radio de Deformación de Rossby (Cushman-Roisin 1986), los cuales predominan en el centro y sur del Sistema de la Corriente del Perú (SCP). Estos frentes son procesos de convergencia de dos masas de agua, y producen una zona de transición de régimen dinámico intenso y de turbulencia geostrófica. Estas características condicionan vórtices o giros anticiclónicos de movimientos de masas de agua y, por la dinámica de Ekman, se estimaría que la acción de los vientos podría afectar hasta una profundidad promedio de 100m (Cushman \& Beckers 2007). Estas caracte- rísticas podrían mantener la productividad marina constante, lo cual favoreceria la presencia de recursos pesqueros.

En la zona centro, la configuración de la plataforma con un ancho mayor a las $60 \mathrm{mn}$ frente a Chimbote jugaría un papel importante, permitiendo que los procesos fotosintéticos se desarrollen plenamente en presencia de las aguas afloradas que llevan gran cantidad de nutrientes y por lo tanto fortalecerían la cadena alimenticia y como consecuencia la concentración de recursos pelágicos. Esta plataforma también se encuentra influenciada por flujos subsuperficiales de origen ecuatorial que transporta agua de la zona ecuatorial (CSPC). Asimismo, por el sur tenemos el subsistema constituido por las Aguas Templadas de la Subantártica (ATSA), al que está asociado el bacalao de profundidad Dissostichus eleginoides y su fauna acompañante.

A estos escenarios se agregan los ambientes litorales en los cuales predominan moluscos, crustáceos y equinodermos de alto valor económico y que sustentan importantes pesquerías artesanales, sobre todo en el sur del Perú.

Variabilidad regional a mediano y largo plazo.- A pesar de encontrarse en la zona tropical, en el mar peruano predominan condiciones templadas a frías que eventualmente son interrumpidas por el fenómeno de El Niño que causa la prevalencia temporal de condiciones tropicales (Fig. 3).

a) Temperatura Superficial del Mar (TSM) .- Las propiedades hidrográficas como la temperatura aportan evidencias de los procesos físicos que modulan su distribución espacial. Las máximas temperaturas ocurren en verano entre las 50 y $100 \mathrm{mn}$ de la costa desde Punta Sal hasta el Callao, y en otońo, desde Punta Negra hasta Supe, mientras que las mínimas se observan en invierno y primavera en las primeras $50 \mathrm{mn}$. La distribución superficial de temperatura (Fig.3) muestra valores mínimos cerca de la costa $(0-50 \mathrm{mn})$ y con una orientación perpendicular a la costa $(E-W)$. Este patrón refleja la importancia del afloramiento 
costero. Los mínimos relativos de temperatura cercanos a la costa se observan a lo largo del año y reflejan aguas subsuperficiales afloradas principalmente en la región sur.

b) Profundidad de la isoterma de $15^{\circ} \mathrm{C}$.- Para el análisis de la variabilidad termal en la vertical, se analizó la profundidad media de la isoterma de $15^{\circ} \mathrm{C}\left(\mathrm{Z}_{15^{\circ} \mathrm{C}}\right)$ como la base de la termoclina, donde en la capa por encima de la termoclina se observaron las variaciones temporales.

La profundidad media de la isoterma de $15^{\circ} \mathrm{C}\left(\mathrm{Z}_{15^{\circ} \mathrm{C}}\right)$ es mayor durante el otońo (abril-junio) en la zona norte y centro,

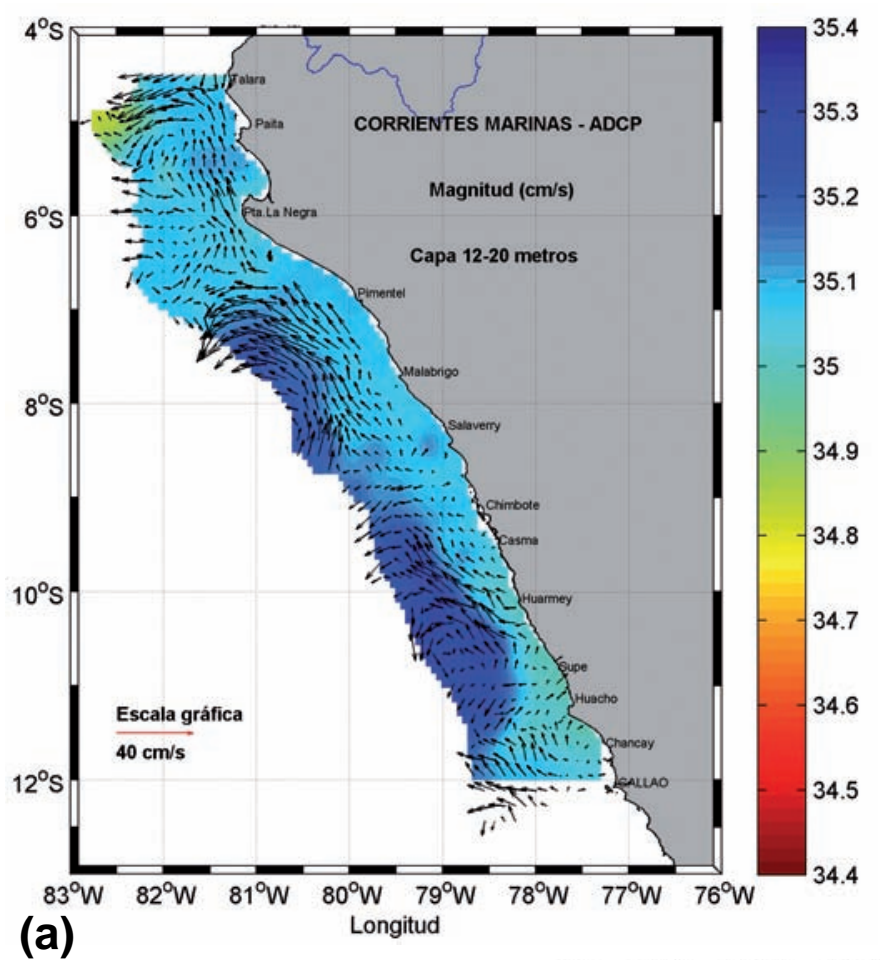

lo cual coincide con la mayor intensificación de la CSPC en la parte norte, que transporta agua cálida con mayor cantidad de oxígeno disuelto de la zona ecuatorial. La menor profundización se presenta en octubre (primavera) cuando se intensifican los afloramientos costeros procedentes del sur. En el sur, en donde los afloramientos costeros son más intensos, la profundidad de la $Z_{15^{\circ} \mathrm{C}}$ es menor (Flores et al. 2009).

En el norte, la $Z_{15^{\circ} \mathrm{C}}$ varía de $108 \mathrm{~m}$ en primavera a $123 \mathrm{~m}$ en otońo, variación significativa debido al incremento de las velocidades de las corrientes subsuperficiales que vienen de la zona ecuatorial. En el centro varía, de $86 \mathrm{~m}$ en invierno a 94 $\mathrm{m}$ en otoño. En el sur, de $60 \mathrm{~m}$ en invierno a $66 \mathrm{~m}$ en verano, presentándose más estable (Fig. 4).

El análisis de la serie de tiempo $(1961$ - 2011) de la profundidad de la isoterma de $15^{\circ} \mathrm{C}\left(Z_{15^{\circ} \mathrm{C}}\right)$ para la zonas norte $(05-$ $\left.08^{\circ} \mathrm{S}\right)$, centro $\left(08-13^{\circ} \mathrm{S}\right)$ y sur $\left(13-18^{\circ} \mathrm{S}\right)$ en el mar peruano, y entre las $50-100 \mathrm{mn}$ (Fig. 5a), revela una alta variabilidad, principalmente en la zona norte, mientras que para toda el área las mayores fluctuaciones se presentan durante los eventos ENSO. En los eventos extraordinarios del fenómeno El Niño de los ańos 1982 - 1983 y 1997 - 1998, los valores promedio de la $Z_{15^{\circ} \mathrm{C}}$ estuvieron entre $140-200 \mathrm{~m}$, mientras que durante los eventos fríos La Nińa fue mucho más superficial y se localizó en alrededor de $30 \mathrm{~m}$. Es decir, durante El Niño, la $Z_{15^{\circ} \mathrm{C}}$ se profundiza en función a la naturaleza y duración del evento, y se hace más superficial durante La Nińa.

$\mathrm{La} \mathrm{Z}_{15^{\circ} \mathrm{C}}$ presentó una tendencia hacia la variabilidad decadal que revela una profundización de dicha isoterma en las décadas de los ochenta y noventa, definiendo un escenario a nivel subsuperficial favorable a los recursos demersales asociados a corrientes de origen ecuatorial como la CSPC (Flores et al.

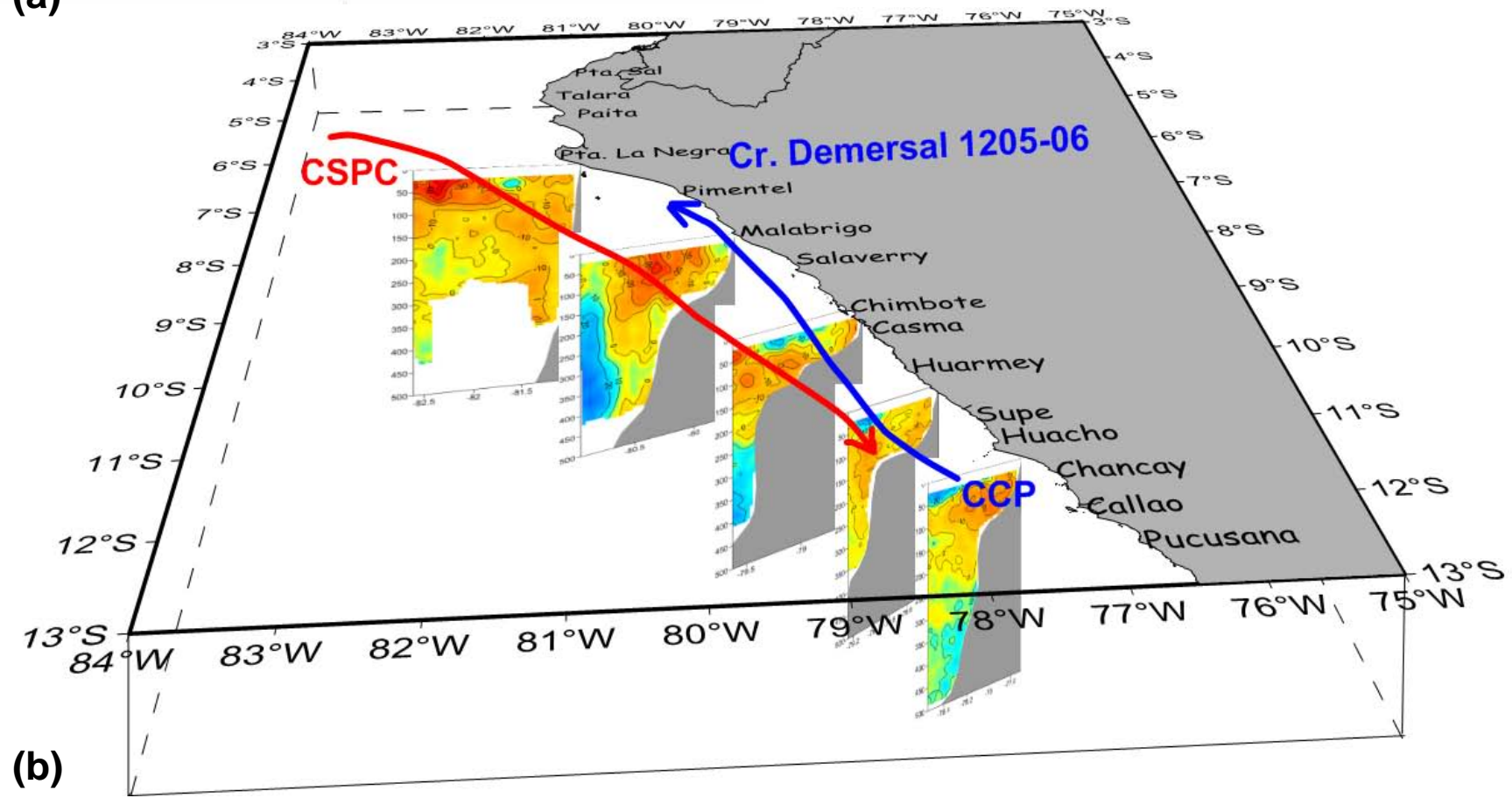

Figura 2. Corrientes marinas obtenidas con ADCP (Acoustic Doppler Current Profiler): (a) Capa vertical integrada entre 12-20m de profundidad (crucero 2012-0910), (b) Secciones verticales (crucero 2012-0506).

Figure 2. Marine currents obtained with ADCP (Acoustic Doppler Current Profiler): (a) Integrated vertical layer from 12-20m depth (cruise 2012-0910), (b) Vertical sections (cruise 2012-0506). 

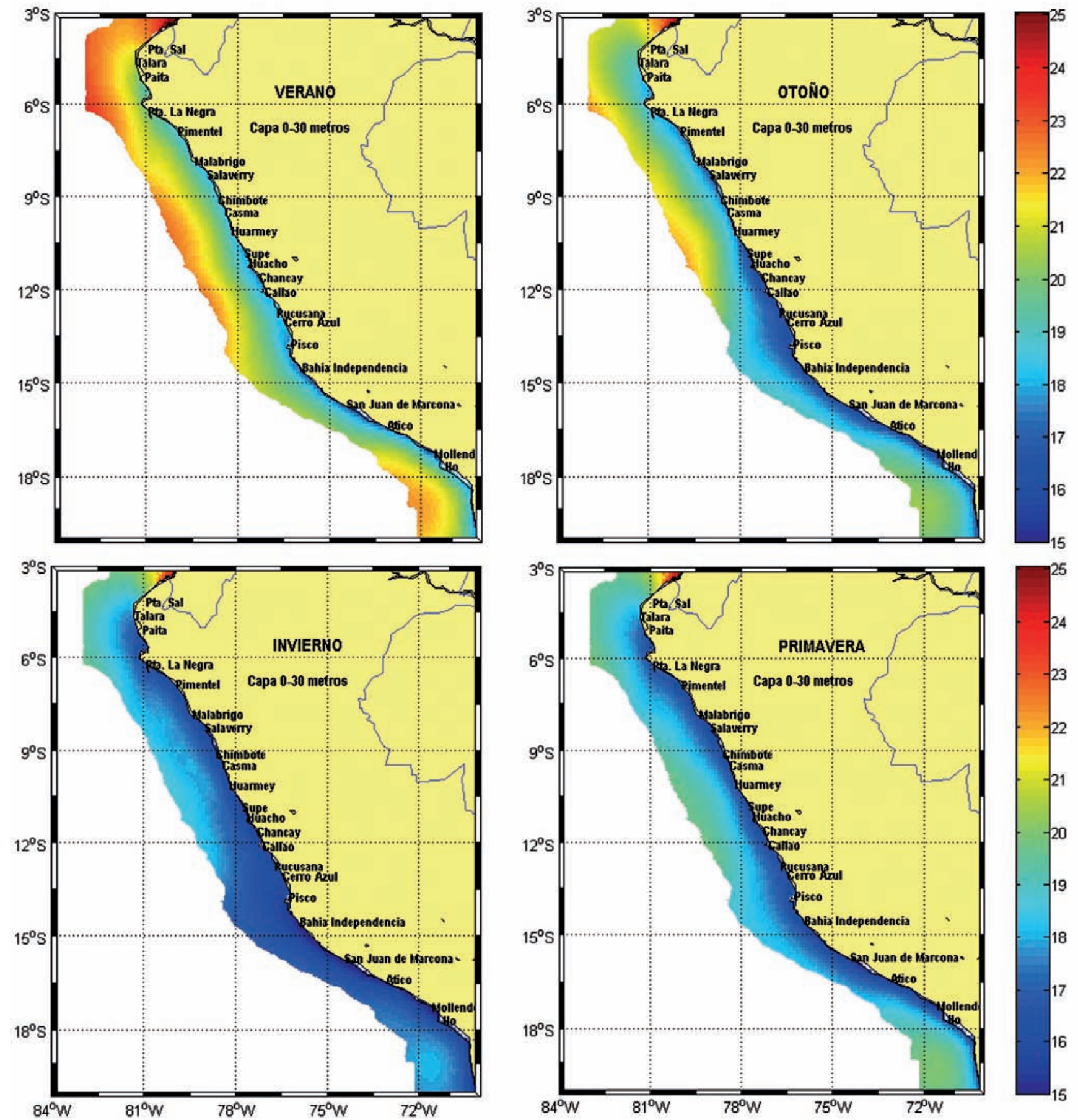

Figura 3. Climatología de la temperatura en la capa de 0-30 m de profundidad entre las 0-100 mn de la costa.

Figure 3. Climatology of temperature in the layer of depth 0-30 m between the 0-100 $\mathrm{mn}$ from the coast.

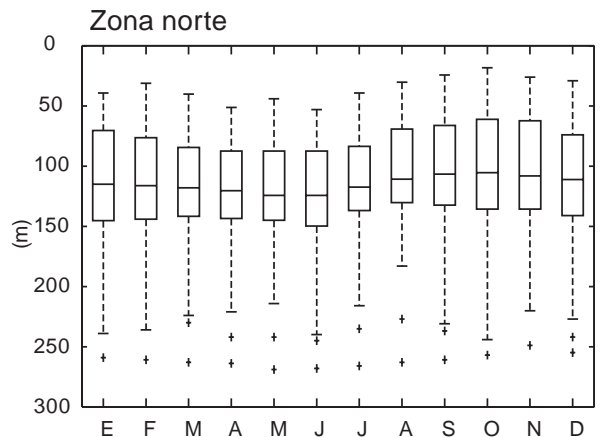

Figura 4. Climatología estacional de la $Z_{15^{\circ} \mathrm{C}}$
Zona centro

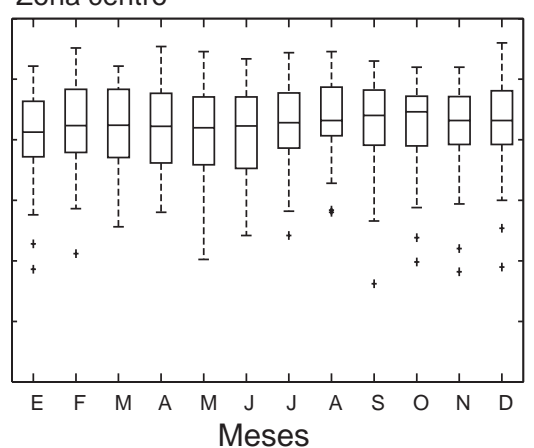

Zona sur

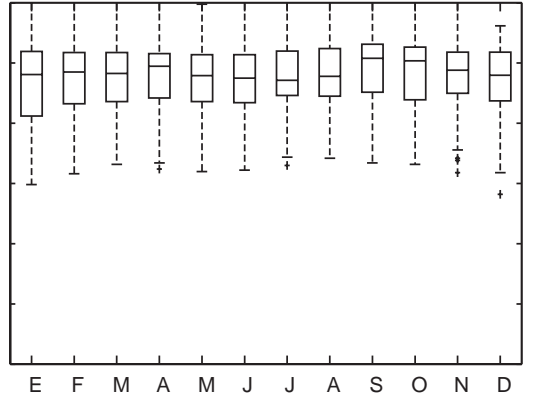

Figure 4. Seasonal climatology of $Z_{15^{\circ} \mathrm{C}}$ 

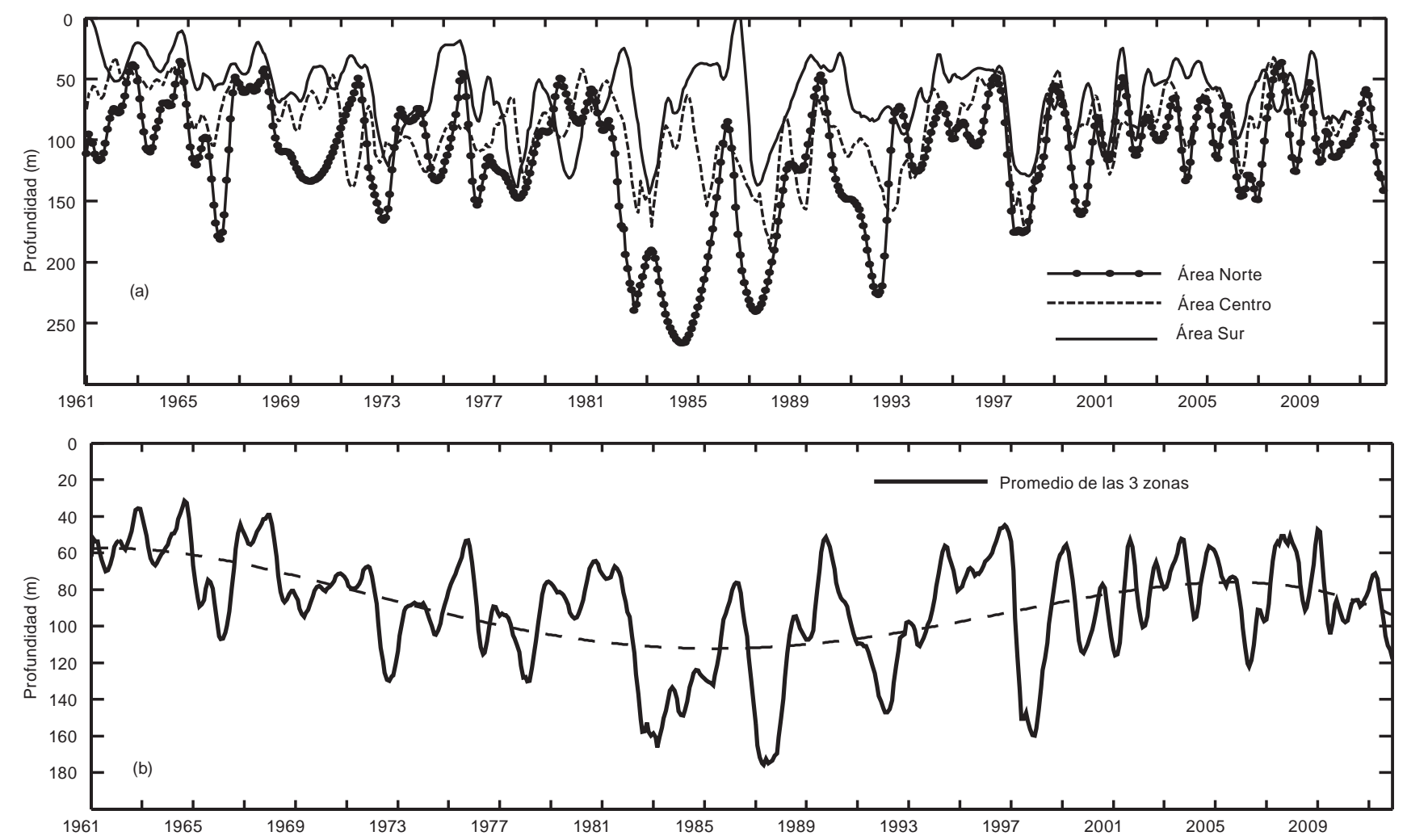

Figura 5. a) Profundidad de la isoterma de $15^{\circ} \mathrm{C}$ de las zonas norte, centro y sur. b) Promedio (norte, centro y sur) de la profundidad de la isoterma de $15^{\circ} \mathrm{C}\left(Z_{15^{\circ} \mathrm{C}}\right)$ entre las $50-100 \mathrm{mn}$ de la costa en el periodo comprendido entre 1961 y 2011.

Figure 5. a) Depth of the $15^{\circ} \mathrm{C}$ isotherm of the northern-center, and southern. b) Average (north, center and south) of $15^{\circ} \mathrm{C}$ isotherm $\left(Z_{15^{\circ} \mathrm{C}}\right)$ depth, between the 50-100 mn from the coast in the period between 1961 and 2011.

2009, Bertrand et al. 2011) (Fig. 5b). Para la zona comprendida entre las $50-100 \mathrm{mn}$ de la costa, la variabilidad de la $Z_{15^{\circ} \mathrm{C}}$ tiene el mismo comportamiento para la zona comprendida entre $0-60 \mathrm{mn}$ de la costa, con ligeras variaciones en las profundidades.

c) Salinidad Superficial del Mar.- Por otro lado, para establecer los patrones de variabilidad de las masas de agua frente al Perú se analizó la serie de la Salinidad Superficial del Mar (SSM) dentro de las $60 \mathrm{mn}$ de la costa para el periodo comprendido entre 1960 y 2008 (Morón 2011). Por lo general, las Aguas Tropicales Superficiales (ATS) y las Aguas Ecuatoriales Superficiales (AES) se mantienen al norte de los 04 y de los $06^{\circ} \mathrm{S}$ respectivamente, proyectándose en cada caso más al sur, hasta aproximadamente los 06 y $08^{\circ} \mathrm{S}$. Especialmente en relación a El Niño (EN) o al arribo de ondas Kelvin, que en muchos casos no generan un evento EN frente a la costa peruana.

En la decada de los sesenta predominaron las salinidades relativamente bajas correspondientes a ACF. En la decada de los setenta hasta mediados de los ochenta se presentó un predominio de ASS, mientras que desde mediados de los ochenta hasta antes de El Niño 1997 - 1998 se presentaron fluctuaciones de ASS y de ACF. En la década del 2000 predominaron ACF (Fig. 6) lo que coincide con lo planteado por Espino (2013).

Las principales manifestaciones de los eventos EN con catego-

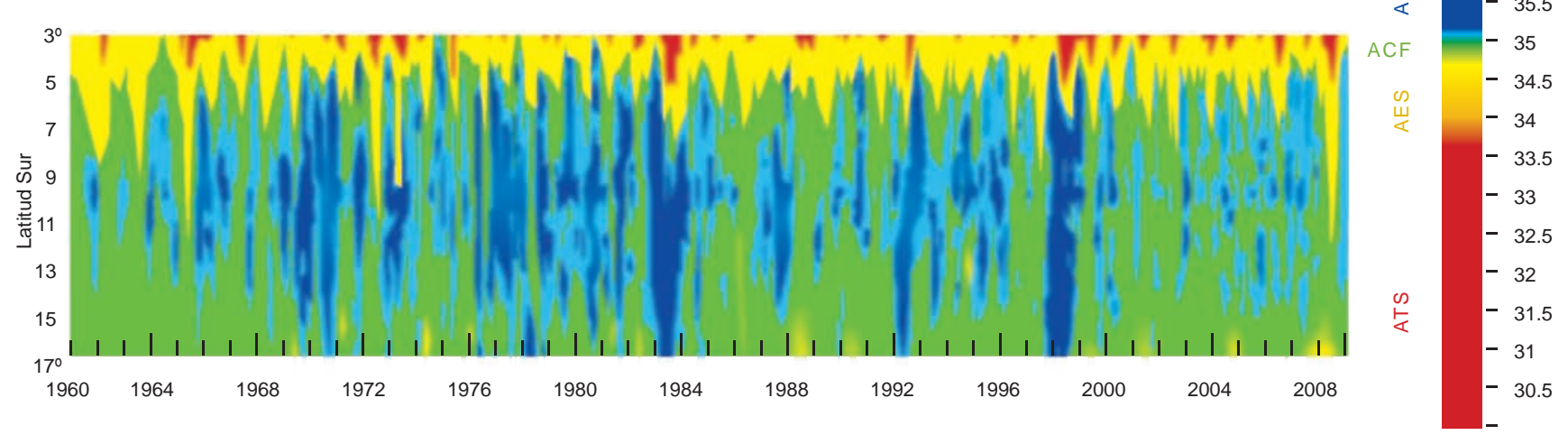

Figura 6. Salinidad superficial del mar entre los 03 y $17^{\circ} \mathrm{S}$ y hasta las $60 \mathrm{mn}$ de la costa en el periodo 1961-2008 (Morón 2011).

Figure 6. Sea surface salinity between 03 and $17^{\circ} \mathrm{S}$ and up to $60 \mathrm{~nm}$ offshore in the period 1961-2008 (Morón 2011). 


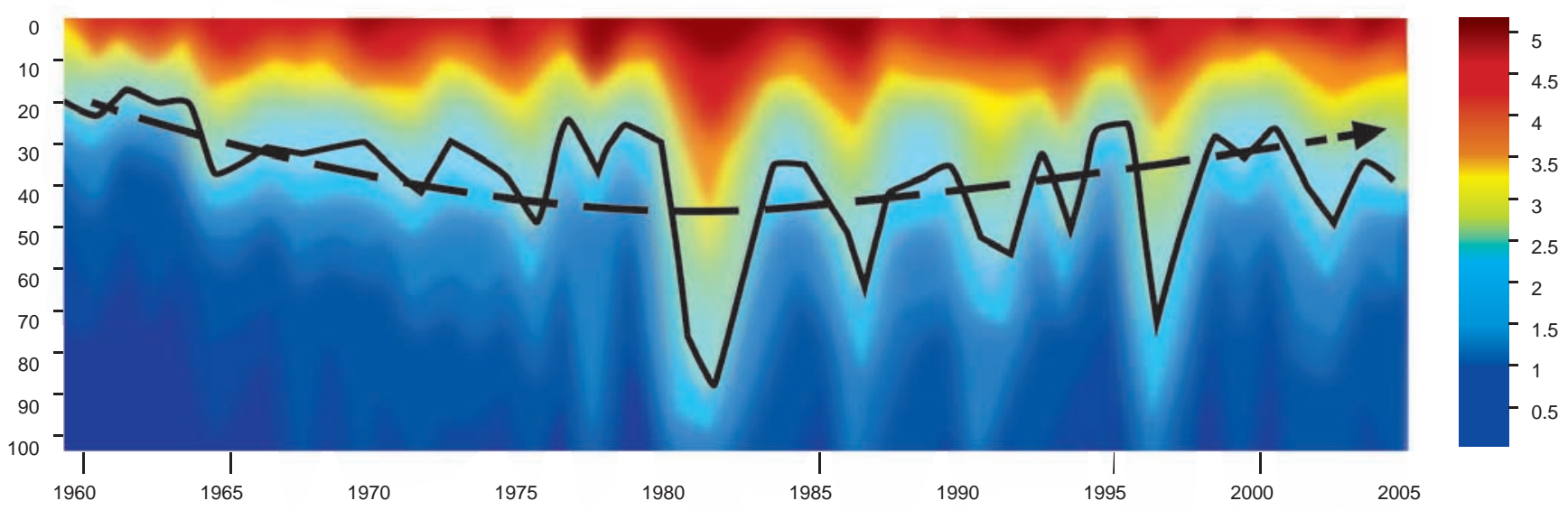

Figura 7. Patrón de variabilidad de la profundidad de la oxiclina $\left(Z_{2 \mathrm{ML} /}\right)($ A. Bertrand, pers. com.).

Figure 7. Variability pattern of the depth of oxycline $\left(Z_{2 \mathrm{ML} /}\right)$ (A. Bertrand, pers. com.).

ría de fuertes o extraordinarios aproximaron a lo largo de la costa ASS de elevada salinidad (35.3 - 35.6). Posteriormente, son las AES y ATS las que se proyectan hacia el sur con baja salinidad, incluso menor a 29.9, lo que da lugar al repliegue hacia el sur de las ASS registradas al inicio del evento, hasta que los vientos del sureste fortalecen los afloramientos y repliegan estas aguas (Zuta et al. 1983, Morón 2011).

d) Profundidad de la oxiclina $\left(\mathrm{Z}_{2 \mathrm{ML} / \mathrm{L}}\right)$.- De acuerdo a Bertrand et al. (2011), la profundidad de la oxiclina $\left(\mathrm{Z}_{2 \mathrm{~m} / \mathrm{L}}\right)$ presentó valores bajos en la década de los sesenta, seguidos de un incremento en la década de los setenta, alcanzando un valor máximo en los años 80 . Estos autores destacan que las mayores profundizaciones se presentaron durante los años El Niño (Fig.7). El valor óptimo de contenido de oxígeno para la presencia de jurel T. murphyi (Dioses 2013) estaría entre 1 y $3 \mathrm{ml} / \mathrm{L}$, por encima de la oxiclina. En las dos últimas décadas (de mil novecientos noventa y de los años dos mil) hubo dominancia de las ACF y la pesca fue más superficial, lo que explicaría las mayores capturas obtenidas en este periodo.

De acuerdo al patrón promedio de captura por áreas de pesca del jurel T. murphyi, las mayores concentraciones en el mar peruano se localizan entre las 50 - $100 \mathrm{mn}$ de la costa (Espinoza et al. 2008). Esta zona se caracteriza por la alta dinámica de los afloramientos costeros y las manifestaciones de los patrones de variabilidad interdecadal (periodos cálidos y fríos), las cuales definen un escenario favorable para el T. murphyi, como sucedió en las décadas de los setenta, ochenta y parte de los noventa. Asimismo, estudios realizados por Dioses (1995) y Grechina et al. (1998) indican que los frentes oceánicos formados por la convergencia de las aguas costeras frías y las aguas subtropicales superficiales serían el hábitat preferente del recurso T. murphyi, lo que se evidencia por las capturas de este recurso. Así mismo Bertrand et al. (2004) refieren que el T. murphyi se ubica en aguas oxigenadas (encima de la oxiclina).

\section{Literatura citada}

Alegre A., P. Espinoza \& M. Espino. 2013. Ecología trófica del jurel Trachurus murphyi en el Perú entre 1977-2011. En: Csirke J., R. GuevaraCarrasco \& M. Espino (Eds.). Ecología, pesquería y conservación del jurel (Trachurus murphyi) en el Perú. Rev. peru. biol. número especial 20(1): $075-082$

Bakun A., \& S. J. Weeks. 2008. The marine ecosystem off Peru: what are the secrets of its fishery productivity and what might its future hold? Progress In Oceanography 79:290 - 299. DOI: 10.1016/j. pocean.2008.10.027
Bárdossy A., 1988. Notes on the robustness of kriging systems. Math. Geology, v. 20, no. 3, pp. 189 - 203. DOI: 10.1007/BF00890253

Bertrand A., M. Segura, M. Gutiérrez \& L. Vásquez. 2004. From small-scale habitat loopholes to decadal cycles: a habitat-based hypothesis explaining fluctuation in pelagic fish populations off Peru. Fish and Fisheries 5: 296 - 316. DOI: 10.1111/j.1467-2679.2004.00165.x

Bertrand A., A. Chaigneau, S. Peraltilla, J. Ledesma \& M. Graco. 2011. Oxygen: A Fundamental Property Regulating Pelagic Ecosystem Structure in the Coastal Southeastern Tropical Pacific. PLoS ONE6(12): e29558. doi:10.1371/ journal.pone. 0029558.

Bougault, G., \& D. Marcotte. 1991. The multivariable variogram and its application to the linear coregionalization model. Journal Mathematical Geology. 23 (10):899 - 928. DOI: 10.1007/BF02066732

Chaigneau, A., Domínguez, N., Eldin, G., Vásquez, L., Flores, R., Grados, C., \& Echevin, V. 2013. Near - coastal circulation in the Northern Humboldt Current System from shipboard ADCP data. Journal of Geophysical Research: Oceans. DOI: 10.1002/jgrc.20328

Chávez F.P., R.T. Barber \& M.P. Sanderson. 1989. The potential Primary Production of the Peruvian Upwelling System. Instituto del Mar del Peru (IMARPE), Deutsche Gesellschaft fur Technische $\mathrm{Zu}$ sammenarbeit (GTZ) and International Center for Living Aquatic Resources Management (ICLARM).

Chávez F.P., A. Bertrand, R. Guevara-Carrasco, P. Soler \& J. Csirke. 2008. The northern Humboldt Current System: Brief history, present status and a view towards the future. En A. Bertrand, R. Guevara-Carrasco, P. Soler, J. Csirke \& F. Chavez (eds.). Progress in Oceanography 79(2 - 4): 95 - 105. DOI: 10.1016/j.pocean.2008.10.012

Cushman-Roisin B. 1986. Frontal geostrophic dynamics. Jounal of Physical Oceanography 16:132-143. DOI: 10.1175/1520-0485(1986)016<0132:FGD>2.0.CO;2

Cushman R.B. \& J.M. Beckers. 2007. Introduction to Geophysical Fluid Dynamics, Academic Press. Pp851

Dioses T. 1995. Análisis de la distribución y abundancia de los recursos jurel y caballa frente a la costa peruana. Informe Progresivo del Instituto del Mar del Perú 3, 55 pp.

Dioses T. 2013. Patrones de distribución y abundancia del jurel Trachurus murphyi en el Perú. En: Csirke J., R. Guevara-Carrasco \& M. Espino (Eds.). Ecología, pesquería y conservación del jurel (Trachurus murphyi) en el Perú. Rev. peru. biol. número especial 20(1): 067 - 074

Espino M. 2003. Estrategia de gestión oriental para el Pacífico Oriental con especial mención a la pesquería peruana. Tesis para optar el grado de Magíster en Geografía con mención en Ordenamiento y Gestión Ambiental. Unidad de Post Grado, Facultad de Ingeniería Geológica, Minera, Metalúrgica y Geográfica, Universidad Nacional Mayor de San Marcos. 102 pp.

Espino M. 2013. El jurel Trachurus murphyi (Nichols 1920) y las variables ambientales de macroescala. En: Csirke J., R. Guevara-Carrasco \& M. Espino (Eds.). Ecología, pesquería y conservación del jurel (Trachurus murphyi) en el Perú. Rev. peru. biol. número especial 20(1): $009-020$

Espino M. y C. Yamashiro. 2012. La variabilidad climática y las pesquerías en el Pacífico suroriental. Lat. Am. J. Aquat. Res., 40(3): 705 - 721 International Conference: "Environment and Resources of the 
South Pacific" P.M. Arana (Guest Editor) DOI: 103856/vol40 issue3 - fulltext -18 .

Espinoza E., M. Niquen \& Flores R. 2008. Changes in the latitudinal and longitudinal distribution of the aggregations of Jack mackerel (Trachurus murphyi) in the Peruvian sea during 1996 - 2007. Chilean Jack Mackerel Workshop 30 June - 4 July 2008.

Flores R., J. Tenorio \& N. Domínguez. 2009. Variaciones de la Extensión Sur de la Corriente Cromwell frente al Perú entre 3 y $14^{\circ} \mathrm{S}$. Bol. Inst. Mar Perú - Callao 24 (1 y 2).

Gaxiola-Castro G, R. Durazo, B. Lavaniegos, M.E. De la Cruz-Orozco, E. Millan-Nunez, L. Soto-Mardones, J. Cepeda-Morales. 2008. Pelagic ecosystem response to interanual variability off Baja California. Cienc. Mar. 34: 263: 26.

Graco, M, J. Ledesma, G. Flores \& M. Girón. 2007. Nutrientes, oxígeno y procesos biogeoquímicos en el sistema de surgencias de la corriente de Humboldt frente a Perú. Revista Peruana de Biología 14 (1): $117-128$

Grechina A., S. Núnez \& D. Arcos. 1998. El desove del recurso jurel, Trachurus symmetricus Murphy (Nichols), en el océano Pacífico Sur. En D. Arcos (Ed.). Biología y ecología del jurel en aguas chilenas. Instituto de Investigación Pesquera, Chile: 117 - 140.

Gutiérrez Torero M. 2002. An approach based in acoustic data to the study of the variability in distribution and abundance of small pelagics in the Humboldt Ecosystem. Ices Symposium on Acoustics in Fisheries and Aquatic Ecology, Montpellier, France 10 - 14 June 2002: 12 p.

Journel A. G. \& C. J. Huijbregts. 1978. Mining Geostatistics. Academic Press. London, pp 600

Montes I., F. Colas, X. Capet \& W. Schneider. 2010. On the pathways of the equatorial subsurface currents in the eastern equatorial Pacific and their contributions to the Peru-Chile Undercurrent. Journal of Geophysical Research: Oceans, 115, C09003, doi:10:1029/2009JC005710.

Morón O. 2000. Características del ambiente marino frente a la costa peruana. Bol. Inst. Mar Perú 19(1-2): 179 - 204 .
Morón O. 2011. Climatología de la salinidad superficial frente a la costa peruana (1960 - 2008), IMARPE 38 (1): 7 - 39.

Rosas Luis R. 2007. Descripción de la alimentación de calamar gigante Dosidicus gigas (D’Orbigny, 1835) en la costa occidental de la península de Baja California. Tesis para obtener el grado de Maestro en Ciencias. Uso, manejo y preservación de los recursos naturales. Orientación: Biología Marina. Centro de Investigaciones Biológicas del Noroeste, S.C. La Paz, Baja California Sur. Septiembre 2007.

Zuta S. \& O.G. Guillén. 1970. Oceanografía de las aguas costeras del Perú. Bol. Inst. Mar Perú (2): 157-324

Zuta S., I. Tsukayama \& R. Villanueva. 1983. El ambiente marino y las fluctuaciones de las principales poblaciones pelágicas de la costa peruana. FAO, informe de pesca 291(2): $179-253$. 